\title{
The Variability in Cytocompatibility and Bone Conduction Based on Different Pore Size and Porosity of n-HA/PA66 Composite Scaffolds
}

\author{
Yiming Liu ${ }^{1+}$, Rui Zhang ${ }^{2}$, Sirui Liu ${ }^{1,3+}$, Jingjing Sun ${ }^{1}$, Xue Zhang ${ }^{1}$, Peng Kang ${ }^{1}$, \\ Ruipu Zhang ${ }^{1}$, Yanyu Yang ${ }^{4}$ and Rui $L^{1}{ }^{1 *}$
}

${ }^{1}$ Department of Stomatology, The First Affiliated Hospital of Zhengzhou University, Zhengzhou, China, ${ }^{2}$ Zhengzhou Stomatology Hospital, Zhengzhou, China, ${ }^{3}$ Academy of Medical Sciences at Zhengzhou University, Zhengzhou, China, ${ }^{4}$ College of Materials Science and Engineering, Zhengzhou University Zhengzhou, Zhengzhou, China

OPEN ACCESS

Edited by:

Elisa Mele,

Loughborough University, United Kingdom

Reviewed by: Pinar Yilgor Huri,

Ankara University, Turkey Saeid Kargozar, Mashhad University of Medical Sciences, Iran

*Correspondence:

Rui $\mathrm{Li}$

fcclir@zzu.edu.cn

${ }^{+}$These authors have contributed equally to this work

Specialty section: This article was submitted to Biomaterials,

a section of the journal

Frontiers in Materials

Received: 04 April 2021

Accepted: 08 July 2021

Published: 12 August 2021

Citation:

Liu Y, Zhang R, Liu S, Sun J, Zhang $X$, Kang $P$, Zhang $R$, Yang $Y$ and $L i R$ (2021) The Variability in

Cytocompatibility and Bone Conduction Based on Different Pore Size and Porosity of n-HAVA66

Composite Scaffolds.

Front. Mater. 8:690732.

doi: 10.3389/fmats.2021.690732
As porous materials, nano-hydroxyapatite/polyamide 66 (n-HA/PA66) composite scaffolds with both desirable bioactivity and good mechanical properties showed great potential to reconstruct the bone defect. Moreover, the pore size and porosity played a key role in the scaffold architecture and cell or bone ingrowth. To investigate the cytocompatibility of different pore size and porosity of n-HA/PA66 composite scaffolds on differentiation and cytocompatibility of osteogenically induced bone marrow-derived mesenchymal stem cells (BMSCs) and bone conduction in repairing the calvarial critical size defect of Sprague-Dawley rats in vivo, we evaluated three kinds of n-HA/PA66 composite scaffolds according to the pore size and porosity in this study (group A: mean pore size was $214 \pm 107.3 \mu \mathrm{m}$, and more than $70 \%$ were arranged in 100-300 $\mu \mathrm{m}$; group B: material mean pore size was $375 \pm 132.2 \mu \mathrm{m}$, and about $60 \%$ were distributed in 300-500 $\mu \mathrm{m}$; group C: mean pore size was $533 \pm 169.4 \mu \mathrm{m}$, and more than $60 \%$ were in 400-700 $\mu \mathrm{m})$. Osteogenically induced BMSCs were seeded in the three types of n-HA PA66 material and cultured in vitro, and the variability on cell adhesion, growth, proliferation, osteogenic differentiation was analyzed using scanning electron microscopy alkaline phosphatase (ALP) and collagen type I (COL I) immunohistochemical staining, as well as quantitative real-time PCR (qRT-PCR) analysis on the osteogenesis-related gene expression (alkaline phosphatase, COL I), was done. Three group matrices/BMSC composites were implanted into the cranial defect of Sprague-Dawley rats. The differentiations of osteogenesis in vivo were then evaluated by histological and qRT-PCR analysis on mRNA levels of OPG and RANKL after 4 and 8 weeks, respectively. The in vitro and in vivo results showed that the group B n-HA/PA66 scaffold was more suitable for osteogenically induced BMSC proliferation, differentiation in vitro, and bone conduction in vivo than groups $A$ and $C$, indicating that the porous $n-H A$ PA66 matrices with a mean pore size of $375 \pm 132.2 \mu \mathrm{m}$ and porosity $77 \pm 2.9 \%$ have better cell biocompatibility and bone conduction.

Keywords: n-HA/PA66 composite, pore size, porosity, biocompatibility, bone conduction 


\section{INTRODUCTION}

Criteria that must be considered in the design of biomaterials include sufficient strength, biocompatibility, appropriate porosity, adequate surface finish guarantee, and serializability (Hulbert et al., 1970; Xi et al., 2020; Yao et al., 2020; Duan et al., 2021). Among these requirements, sufficient strength means the materials have the ability of sufficient modulus of elasticity and a critical stress intensity factor to cope with the patient's increased bone fragility and increased risk of fracture (Li et al., 2021). The mechanical properties of the ideal bone scaffold material should match the host cortical bone, in which the compressive strength of cortical bone is $100-200 \mathrm{Mpa}$ and the modulus of elasticity is 15-25 GPa, while the compressive strength of hydroxyapatite is about $120 \mathrm{MPa}$ and the modulus of elasticity is $75-105 \mathrm{GPa}$, which can match the normal bone cortex (Li et al., 2021; Duan et al., 2020). In addition, pore size and scaffold architecture have to be controlled in order to provide the appropriate scaffold porosity, which encourages cellular activities inside the scaffold and allows nutrients, oxygen, and waste to transport conveniently into or out of the scaffold (Hui et al., 1996; Whang et al., 1999; Hutmacher, 2001; Itala et al., 2001; Ortiz and Jimeno, 2018; Ashrafi et al., 2019; Zhianmanesh et al., 2019). A good material structure can promote better mechanotransduction of cells. Mechanotransduction is the basis for cells to convert mechanical stimuli from the extracellular microenvironment into intracellular biochemical signals to induce a variety of cellular responses and plays an important role in regulating various cellular functions such as development, regeneration, and disease (Song et al., 2020). Many experts have focused on cell or bone ingrowth into porous material with different pore sizes and porosities (Bucholz, 2002; Hollister et al., 2002; Holy et al., 2003; Jones et al., 2006; Otsuki et al., 2006; Kasten et al., 2008; Jones et al., 2009; Bertoldi et al., 2011; Mishra et al., 2012; Ayala et al., 2017; Mbarki et al., 2017). However, the results are still controversial. Mishra et al. found that a threshold permeability of $\sim 3 \times 10-11 \mathrm{~m}^{2}$ was required for vascularization and mineralization to occur within a porous implant, and the pores should be of a critical radius $>50 \mu \mathrm{m}$ (Mishra et al., 2012). However, some researchers considered the cell and bone tissue could grow well in the pores of 50-125 $\mu \mathrm{m}$. Although the accurate pore size and porous volume still remain unknown, the consensus is that the pore size, porosity, and interconnectivity of scaffolds are crucial for cell and bone ingrowth (Hulbert et al., 1970; Gauthier et al., 1998; Lu et al., 1999).

It is well known that hydroxyapatite (HA) has a composition and structure very close to natural bone minerals and therefore has been considered to be the ideal material to build bone tissue engineering scaffold, and polyamide (PA) has already been proved to possess good biocompatibility and excellent mechanical properties (Hutmacher, 2000; Kang et al., 2000; Chu et al., 2002; Kruyt et al., 2003; Bohner and Baumgart, 2004; Uddin et al., 2021; Zalnezhad et al., 2021; Yuan et al., 2021). Our preceding studies fabricated the nano-hydroxyapatite/ polyamide 66 (n-HA/PA66) composite scaffold, which had both desirable bioactivity and good mechanical properties, and

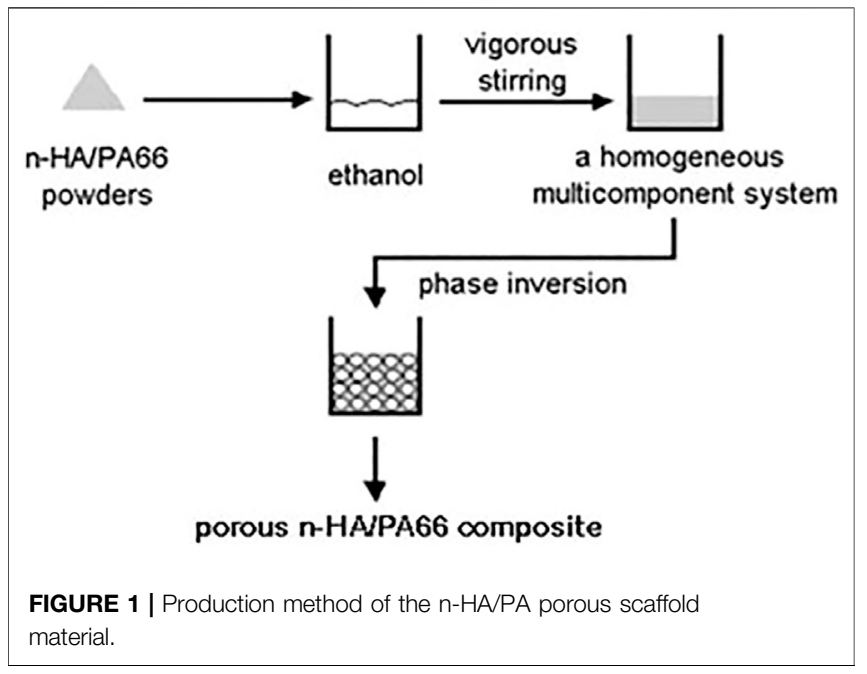

suggested that porous n-HA/PA66 material fulfilled the basic requirements of bone tissue engineering scaffold and had the potential to be applied in orthopedic, reconstructive, and maxillofacial surgery (Marom et al., 2005; Chen et al., 2006; Huang et al., 2007; Wang et al., 2007). Nevertheless, which pore size and porous volume n-HA/PA66 matrices have better cell biocompatibility and bone conduction have not been reported yet. This study investigated the biocompatibility of three different pore sizes and porosity of n-HA/PA66 composite scaffolds (group A: mean pore size was $214 \pm 107.3 \mu \mathrm{m}$, and more than $70 \%$ were arranged in 100-300 $\mu \mathrm{m}$; group B: mean material pore size was $375 \pm 132.2 \mu \mathrm{m}$, and about $60 \%$ were distributed in $300-500 \mu \mathrm{m}$; group C: mean pore size was $533 \pm 169.4 \mu \mathrm{m}$, and more than $60 \%$ were in $400-700 \mu \mathrm{m}$ ) on differentiations and biocompatibility of osteogenically induced bone marrow-derived mesenchymal stem cells (BMSCs) and in vivo osteogenesis in repairing the calvarial critical size defect of Sprague-Dawley rats.

\section{MATERIALS AND METHODS \\ Characterization of n-HA/PA66 Composite Scaffold}

The n-HA/PA porous scaffold was prepared by thermally induced phase inversion. A homogeneous multicomponent system was prepared by mixing n-HA/PA composite powders with ethanol at room temperature. To control the porosity of the resulting porous scaffolds, the composite to ethanol ratio (w:V) varied from 0.6 to 0.8 . After complete mixing, the mixture was cast into the Teflon ${ }^{\circledR}$ mold and moved into ultrasonic vibration for $15 \mathrm{~min}$. Then, the mold was moved into the oven at a temperature of $80^{\circ} \mathrm{C}$ for 3 days. During this period, phase inversion of ethanol was thermally induced by changing the temperature higher than the critical temperature of ethanol. That is, ethanol in the liquid phase changed into a gaseous one. As time passed, the gas nuclei gradually grew bigger and bigger and even interconnected to each other. Following this nucleation and growth mechanism, an interconnective porous 
structure was formed (Chen et al., 2006). Simultaneously, ethanol gradually evaporated from the mold, which leads to the solidification of the material. After complete solidification, the porous foam was removed from the mold and ultrasonic washing in deionized water for $24 \mathrm{~h}$ to thoroughly eliminate the remnants of ethanol. The porous composite scaffold was obtained after being dried at $100^{\circ} \mathrm{C}$. Thereafter, the n-HA/PA scaffolds were airdried in a laminar airflow chamber for $48 \mathrm{~h}$ and sterilized using ethylene oxide gas (Figure 1).

These three pore sizes and porosity of three-dimensional connectivity of the fine porous n-HA/PA66 composites in this study were produced by different ratios of the composite and ethanol. The three kinds of pore size and porosity of the n-HA/ PA66 matrices were fabricated depending on different viscosities of n-HA/PA66 composite slurry by Research Center for NanoBiomaterials, Analytical and Testing Center of Sichuan University (Chen et al., 2006).

\section{Evaluation of the Pores Size and Porosity}

All three materials were cut into $10 \times 10 \times 5 \mathrm{~mm}$ blocks. The three groups of scaffolds were observed under micro-computerized tomography $(\mu-\mathrm{CT})$ with a desktop micro-CT system $(\mu-\mathrm{CT} 80$ scanner, Scanco Medical, Bassersdorf, Switzerland) for the porosities and three-dimensional (3-D) images of three groups of scaffolds. The system was set to $55 \mathrm{kV}, 145 \mathrm{~mA}$, and $500 \mathrm{~ms}$ integration time. The obtained binary images with a resolution of $1024 \times 1024$ pixels and isotropic voxel size of $18 \mu \mathrm{m}$ were reconstructed to $3 \mathrm{D}$ for qualitative and quantitative evaluations (threshold 45-700). The morphologies of the scaffolds were also observed by scanning electronic microscopy (SEM). Prior to the examination, each sample was coated with gold. A JEOL JSM 5600LV SEM microscope at $20 \mathrm{kV}$ was used to perform image analysis. Then, ImageJ soft was used to analyze the mean pore size and distribution of three types of scaffolds.

\section{Evaluation of Mechanical Property}

The compressive strength and modulus of the three groups of composite scaffolds were determined using a mechanical testing machine (Reger-3050, China). Following the guideline of ASTM standard D 695-96, a cylindrical specimen was prepared with a length twice its diameter. In this study, cylindrical samples were prepared with diameters of $10 \mathrm{~mm}$ and lengths of $20 \mathrm{~mm}$. Six porous samples from each group were subjected to this test. The crosshead speed was set at $0.5 \mathrm{~mm} / \mathrm{min}$, and the load was applied until the specimen was compressed to approximately $60 \%$ of its original length. Then, the compressive modulus was calculated.

\section{Assessment of Cell Biocompatibility} Cell Isolation, Culture and Cell Labeling

Bone marrow-derived mesenchymal cells (BMSCs) were isolated under the sterile condition from epiphysis of femur and tibia of the rats weighing an average of $100-120 \mathrm{~g}$ by density gradient centrifugation. After centrifugation, BMSCs were cultured in an incubator under $37^{\circ} \mathrm{C}$ with $5 \%$ humidified $\mathrm{CO}_{2}$. The induced cells were labeled with DiI, as mentioned in our previous study (Boyce and Xing, 2008).
TABLE 1 | Primers used for qRT-PCR.
Gene forward primer sequence $\left(5^{\prime}-3^{\prime}\right)$

ALP TCCCTACCGACCCTGTTCTGA COI-I TCAGAAAAAGCAGCACCG OPG GAAGATCAGCCCAGACGAGATT RANKL AGCGCTTCTCAGGAGTTCCA GAPDH TATGACTCTACCCACGGCAAGT
Reverse primer sequence $\left(5^{\prime}-3^{\prime}\right)$

TGGACCTCTCCCTTGAGTGT TCGTAGCCTTCATAGCCAT TGCTCGCTGGGTTTGCA GCCGGGCCACATCGA

ATACTCAGCACCAGCATCACC

\begin{tabular}{lc}
\hline TABLE 2 | Number of the four group n-HA/PA66 scaffolds in vivo. & \\
\hline Groups & Number \\
\hline Control & 4 \\
A & 6 \\
B & 6 \\
C & 6
\end{tabular}

\section{Flow Cytometry}

For identification of specific cell surface molecules, BMSCs were trypsinized and subsequently incubated with anti-CD29 (1:100), anti-CD31 (1:100), and antibodies (all from, BD Biosciences, Franklin Lakes, NJ, United States) for $1 \mathrm{~h}$, at $4^{\circ} \mathrm{C}$. Cell populations were resolved on an Accuri C6 Flow cytometer (BD Biosciences).

\section{Cell Seeding}

The three types of n-HA/PA66 scaffolds were sterilized using ethylene oxide gas. BMSCs were then seeded onto the tops of the prewetted n-HA/PA66 scaffolds $\left(2.0 \times 10^{5}\right.$ cells/scaffold), and then the scaffold/cell constructs were placed in the wells of tissue culture plates. Seeded scaffolds were incubated with the medium containing osteogenic factors (Dulbecco modified Eagle low-glucose medium containing $10 \%$ FBS, $10 \mathrm{nmol} / \mathrm{L}$ dexamethasones, $10 \mathrm{mmol} / \mathrm{L} \quad \beta$-sodium glycerophosphate, $0.05 \mathrm{mmol} / \mathrm{L} \mathrm{L}$-ascorbic acid) was changed every 3 days.

\section{Observation of the Cell Adhesion and Growth}

The growth information and morphologic changes of cells were observed by an inverted phase-contrast microscope and recorded every day. DiI-labeled cells were observed under a confocal microscope. The adhesion between BMSCs and three groups of n-HA/PA66 was recorded by SEM. Evaluation of proliferation and osteoblastic differentiation: The proliferation of BMSCs on three group scaffolds was determined using (3-\{4,5dimethylthiazol-2yl $\}$-2,5-diphenyl-2H-tetrazoliumbromide)

(MTT) assay as in a previous study (Hutmacher, 2000). The analytical assays were performed in a manner that at least four wells were randomly taken into examination each time.

\section{The Effect of Osteogenic Ability of Cells}

The expression levels of osteogenesis-related genes including ALP, type I collagen (Col- I), OPG, and RANKL were measured using the real-time PCR. Total RNA was isolated from the cells after culturing with n-HA/PA66 scaffold at 4, 7, and 14 days by using Trizol reagents (Invitrogen Life Technologies, Carlsbad, CA, United States) following the manufacturer's protocol. The sequences of primer were listed 

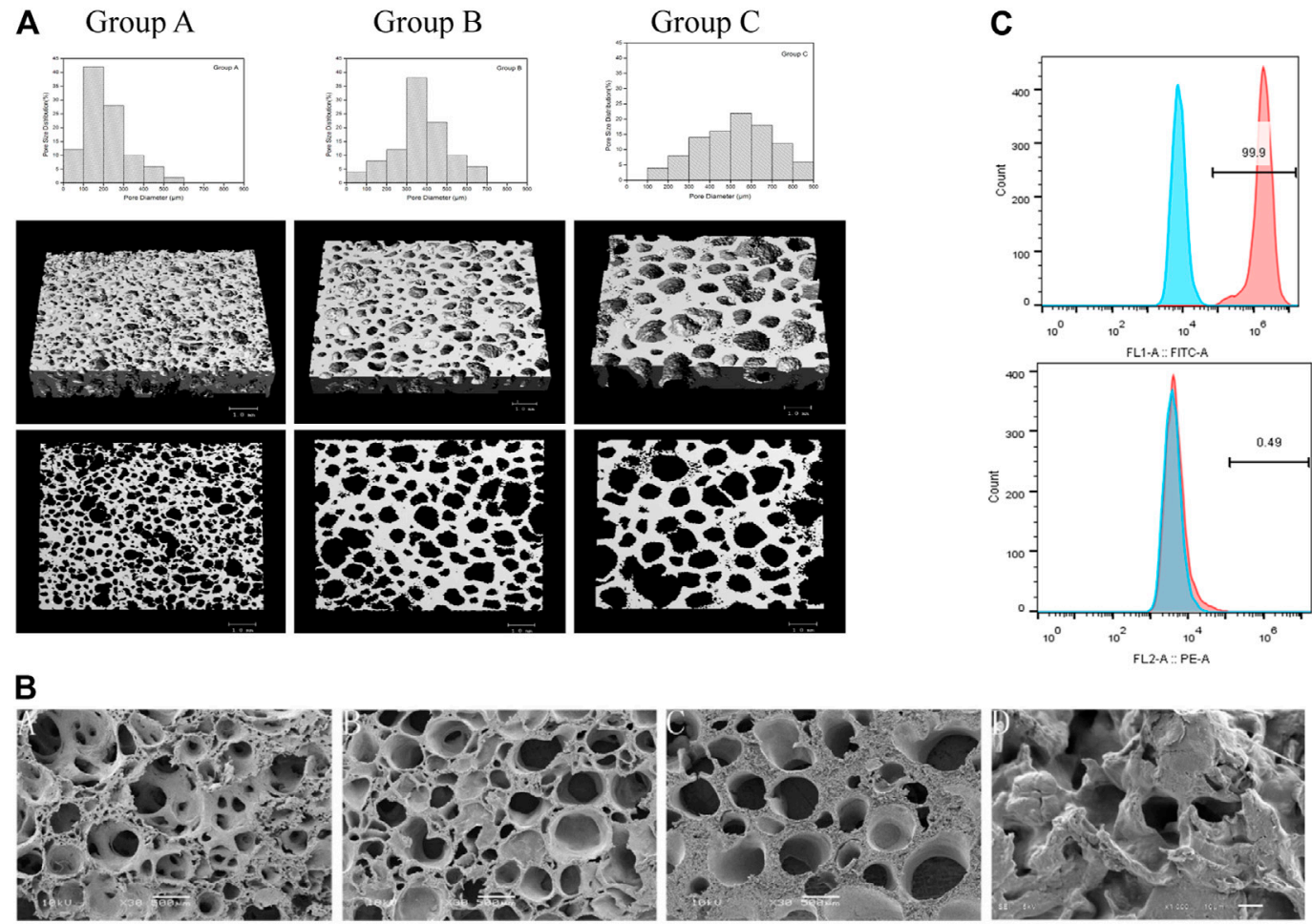

FIGURE 2 | Characterization of n-HA/PA66 composite scaffold. (A) 3D reconstructions of the micro-CT slices illustrating the distinct porosity, pore size, and distribution of the three groups of scaffolds, and the scale bars represent $1 \mathrm{~mm}$. (B) SEM pictures of group A, group B, and group C, each at $\times 500 \mathrm{magnification}$, showing the microporosity of the scaffolds and shows the surface in the $\times 1000$ magnification of the three group scaffolds, whereas no differences were noted. (C) Flow cytometry of BMSCs, including CD29 and CD31.

in Table 1. The expression levels of the target genes were normalized to that of the housekeeping gene GAPDH.

\section{Cytocompatibility and Osteogenesis Post-Implantation In Vivo}

This study was authorized by the Animal Ethics Committee of Zhengzhou University and was carried out in accordance with the Guidelines for the Care and Use of Laboratory Animals. The experimental animals were 40 healthy Sprague-Dawley adult male rats (about 6 weeks old) with an average weight of 300-350 g. The Sprague-Dawley rats were randomly divided into three groups (Table 2): A, B, and C, which corresponded to the three groups of n-HA/PA66 composite scaffolds $(n=36)$; and the last four animals acted as controls whose defects were left empty. The Sprague-Dawley rats were anesthetized via intraperitoneal injection of chloral hydrate. Subsequently, a CSD of $8 \mathrm{~mm}$ diameter was made in the cranium using fissure bur, and implanted according to the corresponding group. Six Sprague-Dawley rats randomly selected from groups A to C were killed for skull samples at 4 and 8 weeks after implantation, respectively. The control group Sprague-Dawley rats were killed only at 8 weeks after implantation. The samples were processed and harvested for the following examinations:

The implants were fixed in $4 \%$ paraformaldehyde, embedded in paraffin, and sliced. The managed specimens were stained with hematoxylin-eosin (H\&E staining) and immunohistochemistry for histologic observation and histomorphometric analysis. Primary antibodies to OPG and RANKL were purchased from Santa Cruz Biotechnology (Santa Cruz, CA, United States). Immunohistochemically staining was performed according to the manufacturer's instructions. Using image analytical software Image-ProPlus (Media Cybernetics, Bethesda, MD), the new bone volume (NBV) was expressed as the percentage of newly formed bone area in the available pore space (bone area/pore area $100 \%$ ).

\section{Statistical Analysis}

Quantitative data are presented as mean (SD). Statistical analysis was assessed using SPSS (version 10.0; SPSS Inc., Chicago, IL). Student $t$-test (assuming equal variances) was performed to determine the statistical significance between experimental groups. $p<0.05$ was considered to be statistically significant.

\section{RESULTS}

\section{Characterization of n-HA/PA66 Composite Scaffold and Bone Marrow-Derived Mesenchymal Stem Cells}

The 3D reconstructed images and microarchitecture of three groups of scaffolds can be observed in Figure 2A; the abundant 
TABLE 3 | Mean pore size, porosity, and mechanical property of the three groups n-HA/PA66 scaffolds.

\begin{tabular}{|c|c|c|c|c|}
\hline Group & Average pore size $(\mu \mathrm{m})$ & Porosity (\%) & Compressive strength (MPa) & Compressive modulus (GPa) \\
\hline$A$ & $214 \pm 107.3$ & $69 \pm 3.8$ & $11.47 \pm 0.18$ & $0.58 \pm 0.04$ \\
\hline$B$ & $375 \pm 132.2^{\star}$ & $77 \pm 2.9^{\star}$ & $7.65 \pm 0.21^{\star}$ & $0.39 \pm 0.06^{\star}$ \\
\hline $\mathrm{C}$ & $533 \pm 169.4^{* \#}$ & $85 \pm 3.2^{* \#}$ & $1.91 \pm 0.09^{* \#}$ & $0.18 \pm 0.03^{* \#}$ \\
\hline
\end{tabular}

*indicates significant difference from group $A(p<0.05)$; \# indicates significant difference from group $B(p<0.05)$.

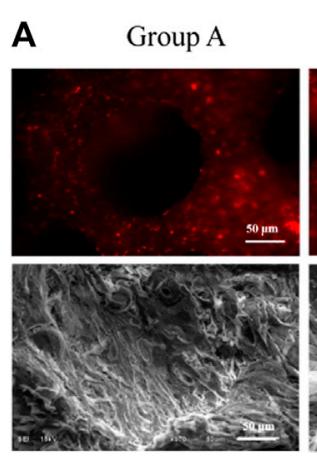

C

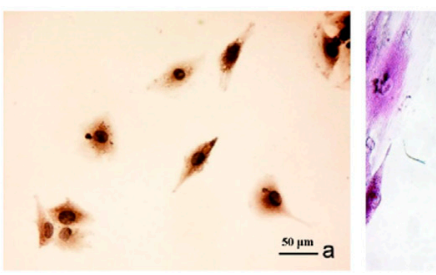

Group B

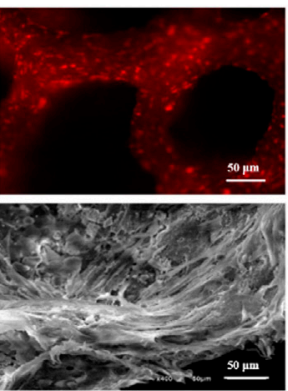

$\underline{s 0 \mu \mathrm{m}}$

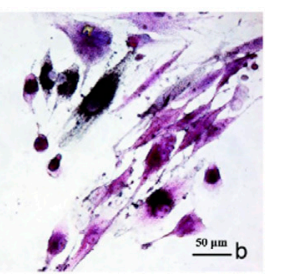

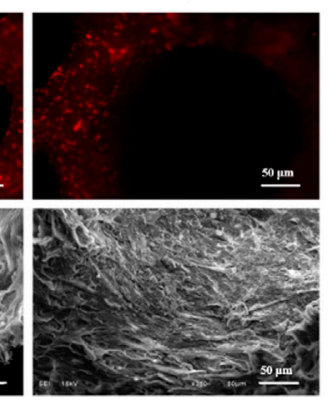

Group C

D
B

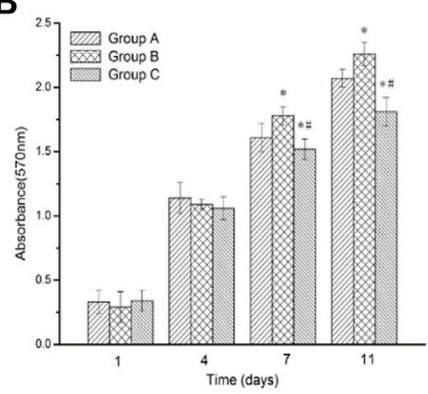

FIGURE 3|Biocompatibility of n-HA/PA66 scaffolds. (A) Fluorescence microscopy and SEM micrographs show the Dil-labeled cells attached to the three groups of n-HA/PA66 scaffolds at 7 days after being seeded in vitro. (B) MTT assay for the proliferation of BMSCs at various incubation periods combined with three types of n-HA/PA66 scaffolds under the same culture condition. (C) Positive ALP staining and Positive COL I staining. (D) Relative mRNA expressions of ALP and Col-1 by BMSCs cultured on the three groups n-HA/PA66 scaffolds for 7 and 14 days * indicates significant difference from group A ( $p<0.05)$; \# indicates significant difference from group B $(p<0.05)$.

interconnections can be noted. The result of SEM (Figure 2B) showed the differences in pore sizes of three scaffolds, but no distinction can be seen under $1000 \times$ magnification. The pore size distribution of each group is shown in Figure 2A. The mean pore size, porosity, compressive strength, and modulus of the three groups composite scaffold are presented in Table 3. The mean pore size of group A scaffold was $214 \pm 107.3 \mu \mathrm{m}$, and above $70 \%$ was arranged in 100-300 $\mu \mathrm{m}$. Group B scaffold's mean pore size was $375 \pm 132.2 \mu \mathrm{m}$, and about $60 \%$ were distributed in $300-500 \mu \mathrm{m}$. The average pore size of group $C$ was $533 \pm 169.4 \mu \mathrm{m}$, and more than $60 \%$ were in $400-700 \mu \mathrm{m}$. The scaffold of group A had the lowest porosity of $69 \pm 3.8 \%$ but the highest mechanical stress, while group $\mathrm{C}$ featured the opposite characteristics.

BMSCs were cultured and detected the origin of the cells by flow cytometry. As the Figure 2C, CD 29 was positive, while CD31 had a negative expression. These results showed that the cells we isolated were mesenchymal.

\section{Cytocompatibility}

The behavior of the mineralization-induced BMSCs cultured with three group scaffolds was observed daily by phase-contrast microscopy. The cells began attaching onto the scaffolds at $24 \mathrm{~h}$, and a large amount of cells proliferated and migrated into the porous material and attached to the wall of the pores after 3 days of culture. Figure 3A shows fluorescence microscopy and SEM micrographs of the three types of scaffolds and it can be observed that there are more cells attaching and growing on group B scaffolds at 7 days.

The MTT assay on the proliferation of BMSCs in contact with three group n-HA/PA66 scaffolds was performed on days 1, 4, 7, and 11 after being cultured. Figure 3B shows the level of group B is higher than groups $\mathrm{A}$ and $\mathrm{C}$ from day $4(p<0.05)$, while the cell population of group $\mathrm{A}$ is more than group $\mathrm{C}$ at 7 and 11 days $(p<$ 0.05 ) (Figure 3B). The positive staining of ALP and COL I immunohistological staining showed that the n-HA/PA66 scaffold, whatever its pore size and porosity, has no negative effects on BMSC osteogenic differentiation (Figure 3C).

The expression levels of osteogenesis-related genes including ALP and COL I were assessed by the quantitative real-time PCR and the results are shown in Figure 3C. Generally, the group B scaffold induced the highest ALP mRNA levels after 7 and 14 days of culture. The relative mRNA level of COL I of group B showed a 


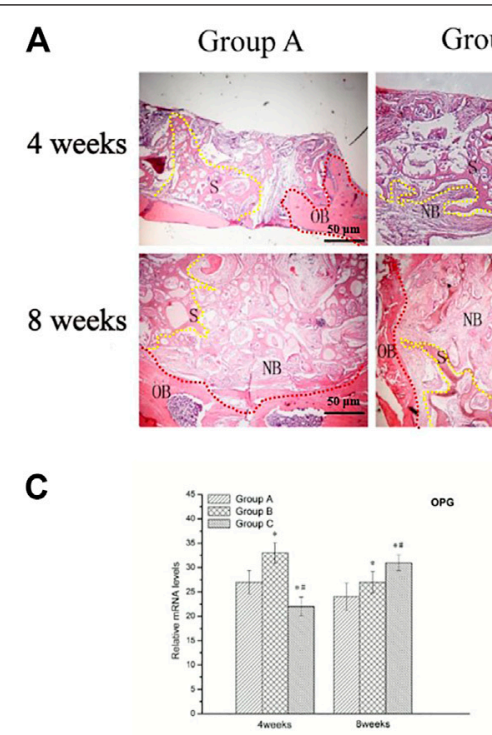

Group B
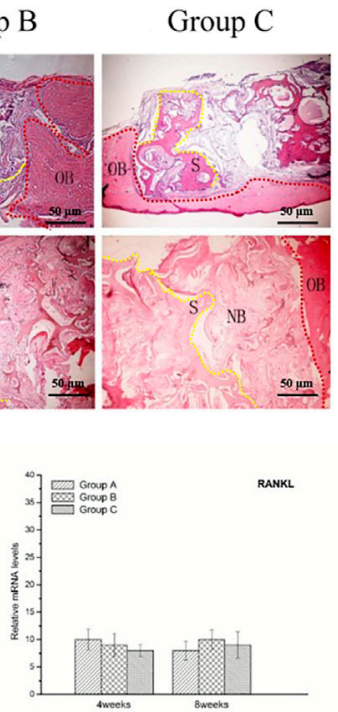

D
B
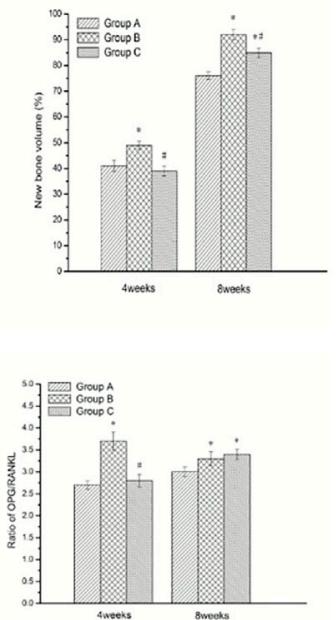

Group C

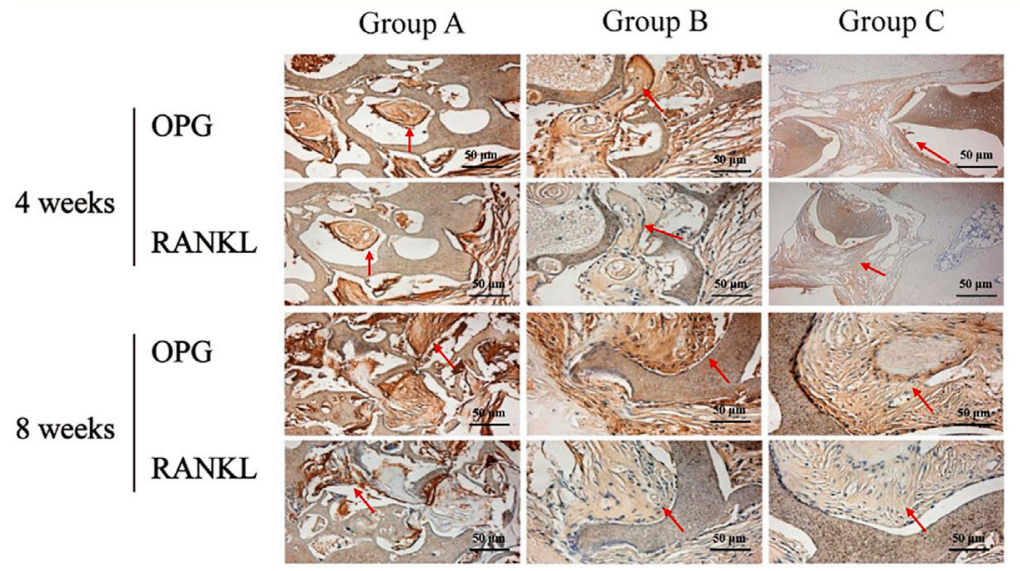

FIGURE 4 | Biocompatibility and bone conduction post-implantation in vivo. (A) Hematoxylin and eosin stained sections of three groups at 4 and 8 weeks postimplantation; NB denotes newly formed bone tissue, S denotes scaffold materials (yellow), while OB denotes original bone (red). And the groups and the observation time are presented in the figure. (B) New bone volume (bone area/pore area $\times 100 \%$ ) of newly formed bone at the bone defect area was repaired using three groups of BMSC/ scaffolds at 4 and 8 weeks after being implanted in vivo. (C) Relative levels of OPG and RANKL mRNA expression, as well as OPG/RANKL ratio in newly formed bone tissue by RT-qPCR, are shown. (D) Immunohistochemical localization and reactivity of RANKL and OPG in the newly formed bone at 4 and 8 weeks after implanted scaffolds (red arrow). * indicates significant difference from group A $(p<0.05)$; \# indicates significant difference from group B $(p<0.05)$.

significant difference with group $\mathrm{A}$ and group $\mathrm{C}$ at 7 days (Figure 3D).

\section{Biocompatibility and Bone Conduction Post-Implantation In Vivo}

At 4 weeks, the histological micrographs (Figure 4A) showed plenty of fibrous connective tissue with minimal mineralization. Although gaps could be seen between the newly formed tissue and the surface of the scaffolds in the three groups, more new tissue and active osteoblasts were observed in group B. At 8 weeks, the boundary between scaffolds and bone was unclear due to the sufficient formation of mature bone tissue, which had grown into the pores and formed a close union without any gap in group $\mathrm{B}$. However, there were not enough new bone regeneration and penetration into the center of scaffold in group $\mathrm{A}$, while in group
C, plenty of gaps were present. In the control group, only fibrous tissues encapsulated the defects.

Quantification of newly formed bone at the bone defect area showed that group B had the highest NBV both at 4 and 8 weeks post-implantation compared to other groups $(p<0.05)$. While no significant differences were showed between group A and group $\mathrm{C}(p>0.05)$ at 4 weeks, the NBV of group $\mathrm{C}$ was higher than group $A$ at 8 weeks $(p<0.05)$ (Figure 3B).

Real-time PCR showed that all three groups had increased expression of OPG and RANKL mRNAs. Especially in specimens with group B scaffold, the relative OPG mRNA levels and the ratio of OPG/RANKL were significantly higher than other groups $(p<0.05)$ at 4 weeks. The result indicated the group B scaffold with proper pore size and porosity might appear to facilitate bone conduction more actively at an early time ( 4 weeks). The result of 8 weeks showed the gene expression of OPG and the ratio of 
OPG/RANKL was decreased in group B, although they still were on a higher level. An increase in OPG expression was noted in group $\mathrm{C} 8$ weeks post-implantation. The images of immunohistochemistry also showed the same trend as with qRT- PCR (Figures 4C,D).

\section{DISCUSSION}

In previous studies, the pores and porosity in porous n-HA/PA66 scaffolds often had a wide range, making it difficult to accurately define the optimum pore size and porosity for cell and bone tissue ingrowth. However, it was hypothesized that in unresorbable materials, the pore size and pore density are equally important (Bodugoz-Senturk et al., 2009). As well as the interconnectivity of pores may be a critical factor for tissue and bone ingrowth. Some researchers determined the importance of interconnections for bone ingrowth and showed that interconnections larger than $50 \mu \mathrm{m}$ were favorable for mineralized bone formation in the interconnections by using hydroxyapatite (HA) and $\beta$-tricalcium phosphate implants. The porosity of the biomaterial plays a significant role in the success of an MSC/scaffold construct (Huang et al., 2011). Most studies (Qu et al., 2011; Uddin et al., 2021; Zalnezhad et al., 2021) showed higher porosity was usually associated with greater bone formation, but the reduction in mechanical properties must be considered.

In our result, the characteristics of the three groups of scaffolds showed evidently different features owing to the slurry of different viscosity used, although they were made by the same matrix composition. Nevertheless, abundant interconnections were seen within the scaffold morphology in all three groups' material. Especially due to their various pore size and porosity, the significant difference in biocompatibility in vitro and in vivo was noted. When BMSCs were cultured with three different pore sizes and porous n-HA/PA66 scaffolds, group B had more cells attaching and growing into its cavity. The result of MTT also showed the population of BMSCs in contact with group B was more than in groups $\mathrm{A}$ and $\mathrm{C}$. It means group $\mathrm{B}$ with a mean pore size $375 \pm 132.2 \mu \mathrm{m}$ and porosity $77 \pm 2.9 \%$ is more suitable for the ingrowth and proliferation of BMSCs. ALP is relied upon by many researchers as the main biochemical marker for osteogenic differentiation and the protein level does not rise in non-induced BMSCs but displays a strong induction during osteogenic differentiation (Zhang et al., 2013). COL I was also considered an important factor in qualitatively determining bone differentiation and mineralization (Feng et al., 2021). The result showed BMSCs in three groups exhibited positive ALP and COL I staining. RT-PCR also revealed that the group B scaffold had a higher level of ALP at 7 and 14 days and COL I at 7 days than other groups. The enhanced level of ALP and COL I expression in this group might indicate the combined effect of osteoblastic differentiation of BMSCs and the group B scaffold, which provides more suitable pore size and porosity.

We implanted BMSC-seeded three groups of n-HA/PA66 scaffolds in the critical cranium defects of S-D rats to confirm the experiment results. Bone regeneration was seen except in the control group. The histological micrographs showed group B constructs showed superior capability in bone regeneration.
The discovery of the RANKL/RANK/OPG system in the mid1990 s in the regulation of bone resorption has led to major advances in our understanding of how bone modeling and remodeling are regulated. RANKL/RANK signaling regulates osteoclast formation, activation, and survival in normal bone modeling and remodeling and in a variety of pathological conditions characterized by increased bone turnover.

OPG protects bone from excessive resorption by binding to RANKL and preventing it from binding to RANK (Li et al., 2016). Thus, RANKL and OPG act as key regulators of bone metabolism and osteoclast biology. OPG/RANKL ratio is an important determinant of bone mass and skeletal integrity (Yao et al., 2016). The relative OPG, RANKL mRNA levels and the ratio of OPG/RANKL were significantly higher in group $B$ than in other groups $(p<0.05)$ at 4 weeks, indicating that the bone conduction in group B was more active.

What is more interesting was that the result at 8 weeks showed the gene expression of OPG and RANKL and the ratio of OPG/ RANKL decreased in groups $A$ and $B$, although they were still on a higher level. In addition, an increase in OPG expression was noted in group $\mathrm{C}$ after implantation for 8 weeks. The images of immunohistochemistry showed the same trend with qRT- PCR, and the statistical analysis on the NBV of group $C$, which was higher than group A at 8 weeks $(p<0.05)$, however, was not identified with the MTT result. A study revealed smaller pores resulted in ingrowth of unmineralized osteoid tissue, and even smaller ones are penetrated only by fibrous tissue. Thus, the same situation was observed in group A. Maybe the bone tissue ingrowth needs a larger pore and interconnectivity size. In comparison, group $\mathrm{C}$, with an average pore size $533 \pm 169.4 \mu \mathrm{m}$ and porosity $85 \pm 3.2 \%$, had a larger pore size which was disadvantageous for more cells attaching onto the scaffold due to its huge lacuna initially but was befitting for bone tissue growth later on than in group A. But the reduced mechanical properties associated with large pore size must be considered. All three groups of n-HA/PA66 scaffolds were produced by the same slurry and the surface structure, the various pore size and porosity affected the biocompatibility and osteogenesis in vitro and in vivo. This is the first study that investigates the effect of pore size and porous volume of n-HA/PA66 matrices on cell biocompatibility and bone conduction, and the results showed that the pore diameter and porosity of group B were more suitable for BMSC proliferation, differentiation in vitro, and bone conduction in vivo.

\section{CONCLUSION}

To detect whether pore size and porous volume n-HA/PA66 matrices have better cell biocompatibility and bone conduction, this study investigated the biocompatibility of three different pore sizes and porosity of n-HA/PA66 composite scaffolds (group A: mean pore size was $214 \pm 107.3 \mu \mathrm{m}$, and more than $70 \%$ were arranged in 100-300 $\mu \mathrm{m}$; group B: mean material pore size was $375 \pm 132.2 \mu \mathrm{m}$, and about $60 \%$ were distributed in $300-500 \mu \mathrm{m}$ and group C: mean pore size was $533 \pm 169.4 \mu \mathrm{m}$, and more than $60 \%$ were in $400-700 \mu \mathrm{m})$ on differentiations and biocompatibility of osteogenically induced bone marrow-derived mesenchymal stem 
cells (BMSCs) and in vivo osteogenesis in repairing the calvarial critical size defect of Sprague-Dawley rats. The results showed that group B material was more suitable for osteogenic-induced BMSC proliferation, differentiation in vitro, and bone conduction in vivo than group A and group C, which indicated that the porous n-HA/ PA66 scaffold with mean pore size $375 \pm 132.2 \mu \mathrm{m}$ and porosity $77 \pm$ $2.9 \%$ has better cell biocompatibility and bone conduction.

\section{DATA AVAILABILITY STATEMENT}

The raw data supporting the conclusions of this article will be made available by the authors without undue reservation.

\section{ETHICS STATEMENT}

The animal study was reviewed and approved by the Medical Ethics Committee of the First Affiliated Hospital of Zhengzhou University.

\section{AUTHOR CONTRIBUTIONS}

YL, RZ (2nd author), and SL have contributed equally to this work. They conceptualized and planned the experiments. YL

\section{REFERENCES}

Ashrafi, Z., Lucia, L., and Krause, W. (2019). Bioengineering Tunable Porosity in Bacterial Nanocellulose Matrices. Soft Matter 15 (45), 9359-9367. doi:10.1039/ c9sm01895f

Ayala, S., Zhang, Z., and Cohen, S. M. (2017). Hierarchical Structure and Porosity in UiO-66 polyMOFs. Chem. Commun. 53 (21), 3058-3061. doi:10.1039/ c6cc10225e

Bertoldi, S., Farè, S., and Tanzi, M. C. (2011). Assessment of Scaffold Porosity: the New Route of Micro-CT. Jabb 9 (3), 165-175. doi:10.5301/JABB.2011.8863

Bodugoz-Senturk, H., Macias, C. E., Kung, J. H., and Muratoglu, O. K. (2009). Poly(vinyl Alcohol)-Acrylamide Hydrogels as Load-Bearing Cartilage Substitute. Biomaterials $30 \quad$ (4), 589-596. doi:10.1016/ j.biomaterials.2008.10.010

Bohner, M., and Baumgart, F. (2004). Theoretical Model to Determine the Effects of Geometrical Factors on the Resorption of Calcium Phosphate Bone Substitutes. Biomaterials 25 (17), 3569-3582. doi:10.1016/ j.biomaterials.2003.10.032

Boyce, B. F., and Xing, L. (2008). Functions of RANKL/RANK/OPG in Bone Modeling and Remodeling. Arch. Biochem. Biophys. 473 (2), 139-146. doi:10.1016/j.abb.2008.03.018

Bucholz, R. W. (2002). Nonallograft Osteoconductive Bone Graft Substitutes. Clin. Orthopaedics Relat. Res. 395, 44-52. doi:10.1097/00003086-200202000-00006

Chen, G., Sircar, K., Aprikian, A., Potti, A., Goltzman, D., and Rabbani, S. A. (2006). Expression of RANKL/RANK/OPG in Primary and Metastatic Human Prostate Cancer as Markers of Disease Stage and Functional Regulation. Cancer 107 (2), 289-298. doi:10.1002/cncr.21978

Chu, T.-M. G., Orton, D. G., Hollister, S. J., Feinberg, S. E., and Halloran, J. W. (2002). Mechanical and In Vivo Performance of Hydroxyapatite Implants with Controlled Architectures. Biomaterials 23 (5), 1283-1293. doi:10.1016/s01429612(01)00243-5

Duan, H., Cao, C., Wang, X., Tao, J., Li, C., Xin, H., et al. (2020). Magnesium-alloy Rods Reinforced Bioglass Bone Cement Composite Scaffolds with Cortical Bone-Matching Mechanical Properties and Excellent Osteoconductivity for performed all the experiments and wrote the manuscript. SL contributed toward editing and proofreading the manuscript. JS and XZ performed the experiments of immunofluorescence staining. $\mathrm{Pk}, \mathrm{RZ}$ (7th author), and YY performed the experiments of quantitative polymerase chain reaction. RL was responsible for the overall project design and manuscript organization, revision, and finalization.

\section{FUNDING}

This study was funded by the National Natural Science Foundation of China (31670994, U1904145, 81901039), Nature Science Foundation of Henan province (182300410340), and the Union Project of Medical and Technology Research Program of Henan Province (LHGJ20190191, SBGJ20203047, SBGJ202002073). They all performed the responsibility of conceptualization, Methodology, Software, and the preparation of writing the draft.

\section{ACKNOWLEDGMENTS}

We thank the Academy of Medical Sciences at Zhengzhou University and Sichuan University for providing the experimental facilities and laboratory space.

Load-Bearing Bone In Vivo Regeneration. Sci. Rep. 10 (1), 18193. doi:10.1038/ s41598-020-75328-7

Duan, H., Chen, X., Wu, Y., Leng, Y., Huang, X., and Xiong, Y. (2021). Integrated Nanoparticle Size with Membrane Porosity for Improved Analytical Performance in sandwich Immunochromatographic Assay. Analytica Chim. Acta 1141, 136-143. doi:10.1016/j.aca.2020.10.041

Feng, C., Yao, J., Wang, L., Zhang, X., and Fan, Y. (2021). Idealized Conductance: A New Method to Evaluate Stiffness of Trabecular Bone. Int. J. Numer. Meth Biomed. Engng 37 (3), e3425. doi:10.1002/cnm.3425

Gauthier, O., Bouler, J. M., Aguado, E., Pilet, P., and Daculsi, G. (1998). Macroporous Biphasic Calcium Phosphate Ceramics: Influence of Macropore Diameter and Macroporosity Percentage on Bone Ingrowth. Biomaterials 19 (1-3), 133-139. doi:10.1016/s0142-9612(97)00180-4

Hollister, S. J., Maddox, R. D., and Taboas, J. M. (2002). Optimal Design and Fabrication of Scaffolds to Mimic Tissue Properties and Satisfy Biological Constraints. Biomaterials 23 (20), 4095-4103. doi:10.1016/s0142-9612(02) 00148-5

Holy, C. E., Fialkov, J. A., Davies, J. E., and Shoichet, M. S. (2003). Use of a Biomimetic Strategy to Engineer Bone. J. Biomed. Mater. Res. 65A (4), 447-453. doi:10.1002/jbm.a.10453

Huang, D., Zuo, Y., Zou, Q., Zhang, L., Li, J., Cheng, L., et al. (2011). Antibacterial Chitosan Coating on Nano-Hydroxyapatite/polyamide66 Porous Bone Scaffold for Drug Delivery. J. Biomater. Sci. Polym. Edition 22 (7), 931-944. doi:10.1163/ $092050610 X 496576$

Huang, J., Lin, Y. W., Fu, X. W., Best, S. M., Brooks, R. A., Rushton, N., et al. (2007). Development of Nano-Sized Hydroxyapatite Reinforced Composites for Tissue Engineering Scaffolds. J. Mater. Sci. Mater. Med. 18 (11), 2151-2157. doi:10.1007/s10856-007-3201-8

Hui, P. W., Leung, P. C., and Sher, A. (1996). Fluid Conductance of Cancellous Bone Graft as a Predictor for Graft-Host Interface Healing. J. Biomech. 29 (1), 123-132. doi:10.1016/0021-9290(95)00010-0

Hulbert, S. F., Young, F. A., Mathews, R. S., Klawitter, J. J., Talbert, C. D., and Stelling, F. H. (1970). Potential of Ceramic Materials as Permanently Implantable Skeletal Prostheses. J. Biomed. Mater. Res. 4 (3), 433-456. doi:10.1002/jbm.820040309 
Hutmacher, D. W. (2001). Scaffold Design and Fabrication Technologies for Engineering Tissues - State of the Art and Future Perspectives. J. Biomater. Sci. Polym. Edition 12 (1), 107-124. doi:10.1163/156856201744489

Hutmacher, D. W. (2000). Scaffolds in Tissue Engineering Bone and Cartilage. Biomaterials 21 (24), 2529-2543. doi:10.1016/s0142-9612(00)00121-6

Itälä, A. I., Ylänen, H. O., Ekholm, C., Karlsson, K. H., and Aro, H. T. (2001). Pore Diameter of More Than $100 \mu \mathrm{m}$ Is Not Requisite for Bone Ingrowth in Rabbits. J. Biomed. Mater. Res. 58 (6), 679-683. doi:10.1002/jbm.1069

Jones, A. C., Arns, C. H., Hutmacher, D. W., Milthorpe, B. K., Sheppard, A. P., and Knackstedt, M. A. (2009). The Correlation of Pore Morphology, Interconnectivity and Physical Properties of 3D Ceramic Scaffolds with Bone Ingrowth. Biomaterials 30 (7), 1440-1451. doi:10.1016/ j.biomaterials.2008.10.056

Jones, J. R., Ehrenfried, L. M., and Hench, L. L. (2006). Optimising Bioactive Glass Scaffolds for Bone Tissue Engineering. Biomaterials 27 (7), 964-973. doi:10.1016/j.biomaterials.2005.07.017

Kang, M. I., Lee, W. Y., Oh, K. W., Han, J. H., Song, K. H., Cha, B. Y., et al. (2000). The Short-Term Changes of Bone mineral Metabolism Following Bone Marrow Transplantation. Bone 26 (3), 275-279. doi:10.1016/s8756-3282(99)00265-3

Kasten, P., Beyen, I., Niemeyer, P., Luginbühl, R., Bohner, M., and Richter, W. (2008). Porosity and Pore Size of $\beta$-tricalcium Phosphate Scaffold Can Influence Protein Production and Osteogenic Differentiation of Human Mesenchymal Stem Cells: An In Vitro and In Vivo Study. Acta Biomater. 4 (6), 1904-1915. doi:10.1016/j.actbio.2008.05.017

Kruyt, M. C., de Bruijn, J. D., Wilson, C. E., Oner, F. C., van Blitterswijk, C. A., Verbout, A. J., et al. (2003). Viable Osteogenic Cells Are Obligatory for TissueEngineered Ectopic Bone Formation in Goats. Tissue Eng. 9 (2), 327-336. doi:10.1089/107632703764664792

Li, J., You, F., Li, Y., Zuo, Y., Li, L., Jiang, J., et al. (2016). Bone Regeneration and Infiltration of an Anisotropic Composite Scaffold: an Experimental Study of Rabbit Cranial Defect Repair. J. Biomater. Sci. Polym. Edition 27 (4), 327-338. doi:10.1080/09205063.2015.1128248

Li, S., Wang, T., Hu, J., Li, Z., Wang, B., Wang, L., et al. (2021). Surface Porous PolyEther-Ether-Ketone Based on Three-Dimensional Printing for Load-Bearing Orthopedic Implant. J. Mech. Behav. Biomed. Mater. 120, 104561. doi:10.1016/ j.jmbbm.2021.104561

Lu, J. X., Flautre, B., Anselme, K., Hardouin, P., Gallur, A., Descamps, M., et al. (1999). Role of Interconnections in Porous Bioceramics on Bone Recolonization In Vitro and In Vivo. J. Mater. Sci. Mater. Med. 10 (2), 111-120. doi:10.1023/a:1008973120918

Marom, R., Shur, I., Solomon, R., and Benayahu, D. (2005). Characterization of Adhesion and Differentiation Markers of Osteogenic Marrow Stromal Cells. J. Cel. Physiol. 202 (1), 41-48. doi:10.1002/jcp.20109

Mbarki, M., Sharrock, P., Fiallo, M., and Elfeki, H. (2017). Hydroxyapatite Bioceramic with Large Porosity. Mater. Sci. Eng. C 76, 985-990. doi:10.1016/j.msec.2017.03.097

Mishra, S., Rajyalakshmi, A., and Balasubramanian, K. (2012). Compositional Dependence of Hematopoietic Stem Cells Expansion on Bioceramic Composite Scaffolds for Bone Tissue Engineering. J. Biomed. Mater. Res. 100 (9), 2483-2491. doi:10.1002/jbm.a.34145

Ortiz, F., and Jimeno, E. (2018). Analysis of the Porosity of Endodontic Sealers through Micro-computed Tomography: A Systematic Review. J. Conserv Dent 21 (3), 238-242. doi:10.4103/JCD.JCD_346_17

Otsuki, B., Takemoto, M., Fujibayashi, S., Neo, M., Kokubo, T., and Nakamura, T. (2006). Pore Throat Size and Connectivity Determine Bone and Tissue Ingrowth into Porous Implants: Three-Dimensional Micro-CT Based Structural Analyses of Porous Bioactive Titanium Implants. Biomaterials 27 (35), 5892-5900. doi:10.1016/j.biomaterials.2006.08.013

Qu, D., Li, J., Li, Y., Khadka, A., Zuo, Y., Wang, H., et al. (2011). Ectopic Osteochondral Formation of Biomimetic Porous PVA-n-HA/PA6 Bilayered
Scaffold and BMSCs Construct in Rabbit. J. Biomed. Mater. Res. 96B (1), 9-15. doi:10.1002/jbm.b.31697

Song, Y., Soto, J., Chen, B., Yang, L., and Li, S. (2020). Cell Engineering: Biophysical Regulation of the Nucleus. Biomaterials 234, 119743. doi:10.1016/ j.biomaterials.2019.119743

Uddin, M., Hall, C., Santos, V., Visalakshan, R., Qian, G., and Vasilev, K. (2021). Synergistic Effect of Deep ball Burnishing and HA Coating on Surface Integrity, Corrosion and Immune Response of Biodegradable AZ31B Mg Alloys. Mater. Sci. Eng. C 118, 111459. doi:10.1016/j.msec.2020.111459

Wang, H., Li, Y., Zuo, Y., Li, J., Ma, S., and Cheng, L. (2007). Biocompatibility and Osteogenesis of Biomimetic Nano-Hydroxyapatite/polyamide Composite Scaffolds for Bone Tissue Engineering. Biomaterials 28 (22), 3338-3348. doi:10.1016/j.biomaterials.2007.04.014

Whang, K., Healy, K. E., Elenz, D. R., Nam, E. K., Tsai, D. C., Thomas, C. H., et al. (1999). Engineering Bone Regeneration with Bioabsorbable Scaffolds with Novel Microarchitecture. Tissue Eng. 5 (1), 35-51. doi:10.1089/ ten.1999.5.35

Xi, J., Li, H., Xi, J., Tan, S., Zheng, J., and Tan, Z. (2020). Preparation of High Porosity Biochar Materials by Template Method: a Review. Environ. Sci. Pollut. Res. 27 (17), 20675-20684. doi:10.1007/s11356-020-08593-8

Yao, M.-Z., Huang-Fu, M.-Y., Liu, H.-N., Wang, X.-R., Sheng, X., and Gao, J.-Q. (2016). Fabrication and Characterization of Drug-Loaded NanoHydroxyapatite/polyamide 66 Scaffolds Modified with Carbon Nanotubes and Silk Fibroin. Ijn Vol. 11, 6181-6194. doi:10.2147/IJN.S106929

Yao, M. S., Zheng, J. J., Wu, A. Q., Xu, G., Nagarkar, S. S., Zhang, G., et al. (2020). A Dual-Ligand Porous Coordination Polymer Chemiresistor with Modulated Conductivity and Porosity. Angew. Chem. Int. Ed. 59 (1), 172-176. doi:10.1002/anie.201909096

Yuan, X., Ding, L., Diao, J., Wen, S., Xu, C., Zhou, L., et al. (2021). PolyMetHA Nanocomplexs Regulates Glucose Uptake by Inhibiting SHIP2 Activity. J. Biomater. Appl. 35 (7), 849-856. doi:10.1177/ 0885328220947343

Zalnezhad, E., Musharavati, F., Chen, T., Jaber, F., Uzun, K., Chowdhury, M. E. H., et al. (2021). Tribo-mechanical Properties Evaluation of HA/TiO2/CNT Nanocomposite. Sci. Rep. 11 (1), 1867. doi:10.1038/s41598-021-81187-7

Zhang, F., Wu, J., Kang, D., and Zhang, H. (2013). Development of a Complex Hydrogel of Hyaluronan and PVA Embedded with Silver Nanoparticles and its Facile Studies on Escherichia coli. J. Biomater. Sci. Polym. Edition 24 (12), 1410-1425. doi:10.1080/09205063.2012.763109

Zhianmanesh, M., Varmazyar, M., and Montazerian, H. (2019). Fluid Permeability of Graded Porosity Scaffolds Architectured with Minimal Surfaces. ACS Biomater. Sci. Eng. 5 (3), 1228-1237. doi:10.1021/acsbiomaterials.8b01400

Conflict of Interest: The authors declare that the research was conducted in the absence of any commercial or financial relationships that could be construed as a potential conflict of interest.

Publisher's Note: All claims expressed in this article are solely those of the authors and do not necessarily represent those of their affiliated organizations, or those of the publisher, the editors and the reviewers. Any product that may be evaluated in this article, or claim that may be made by its manufacturer, is not guaranteed or endorsed by the publisher.

Copyright $\odot 2021$ Liu, Zhang, Liu, Sun, Zhang, Kang, Zhang, Yang and Li. This is an open-access article distributed under the terms of the Creative Commons Attribution License (CC BY). The use, distribution or reproduction in other forums is permitted, provided the original author(s) and the copyright owner(s) are credited and that the original publication in this journal is cited, in accordance with accepted academic practice. No use, distribution or reproduction is permitted which does not comply with these terms. 